\title{
Impacted Esophageal Foreign Body Mistaken for Chronic Pharyngitis
}

An otherwise healthy well-thriving 18-mo-old boy was brought to pediatric emergency department with complaints of intermittent fever, vomiting and dysphagia for solid foods for last 3 months. He was treated as chronic pharyngitis at several hospitals before referral, and no chest radiograph was obtained before. Examination revealed congested posterior pharyngeal wall, bilateral normal air entry and no added chest sounds. Chest radiograph revealed a coiled spring impacted in upper esophagus (Fig. 1), which was removed endoscopically. After 48 hours, child was discharged. On one month follow up, patient was asymptomatic and doing well.

Diagnosis of gastrointestinal foreign bodies is straightforward, if caregivers witness a child taking any foreign body in mouth. Otherwise, it can lead to delay in diagnosis as patient may present with non-specific symptoms viz., unexplained fever, sore throat, cough, stridor, wheezing, vomiting, dysphagia and abdominal pain generating large number of differential diagnoses. ${ }^{1,2}$ Hence gastrointestinal foreign body should be suspected in any healthy child visiting physician repeatedly for aforementioned symptoms which are for long duration and not improving with standard treatment for more prevalent conditions. ${ }^{3}$ Dysphagia should be suggestive of esophageal foreign body.

Ethical Approval: Retrospective case reports do not need approval from Institute Ethics Committee as per our Institute's policy.

Funding : None

Competing Interests: None

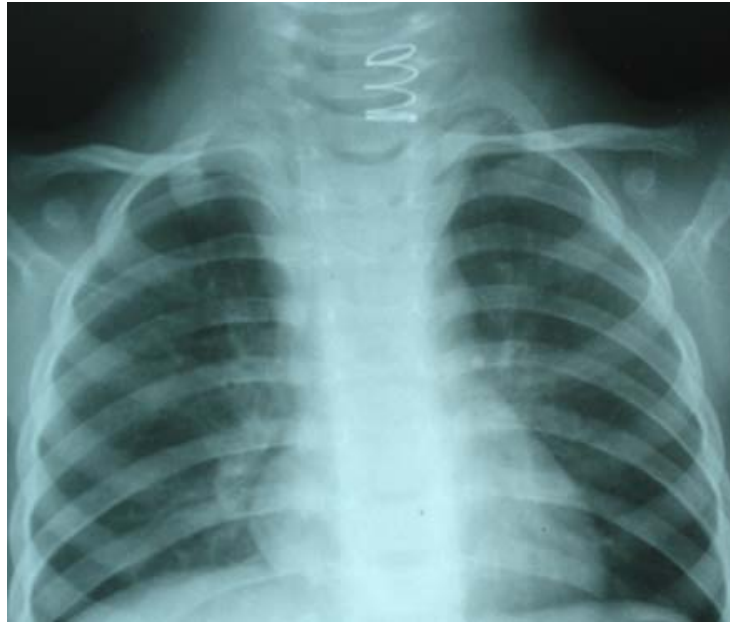

Fig. 1. Impacted foreign body in upper esophagus.

G.M. Narasimhappa, Arun K. Baranwal and Sunit C. Singhi

Emergency and Critical Care Division, Department of

Pediatrics, Advanced Pediatrics Center, Postgraduate Institute of Medical Education and Research, Chandigarh,

India

E-mail: sunit.singhi@gmail.com [DOI-10.1007/s12098-009-0152-8]

\section{REFERENCES}

1. Roderick IM, Jeanne GH, Biemann O, Edward PT, Charles DS. Esophageal foreign bodies in children: diagnosis, treatment and complications. AJR 1996; 166: 919-924.

2. Gregory P, James AH. Esophageal coin with unusual radiologic appearance. Pediatr Emerg Care 2005; 21: 667-669.

3. Louie JP, Alpern ER, Windreich RM. Witnessed and unwitnessed esophageal foreign bodies in children. Pediatr Emerg Care 2005; 21: 582-585. 